\title{
Descrição da Larva de Último Estádio de Oxyagrion sulmatogrossense Costa, Souza \& Santos (Odonata, Coenagrionidae)
}

\author{
Marina Schmidt Dalzochio ${ }^{1} \&$ Marciel Élio Rodrigues ${ }^{2}$
}

Agência de Fomento: Conselho Nacional Desenvolvimento Científico e Tecnológico (CNPq). 1. Pesquisadora autônoma. Rua Terezina, 2305 - Bairro Tropical, CEP 85807-140 - Cascavel-PR-Brasil. e-mail: mahsdalzochio@gmail.com. Autor para correspondência. 2. Universidade Estadual do Oeste do Paraná (UNIOESTE), e-mail: marcielelio@uol.com.br

EntomoBrasilis 2 (3): 73-75(2009)

Resumo. A larva de último ínstar de Oxyagrion sulmatogrossense Costa, Souza \& Santos 2000 é descrita e ilustrada a partir de material procedente de Cascavel, Paraná, Brasil.

Palavras-Chaves: Imaturos, Morfologia, Sistemática, Zygoptera.

\section{Description of the last instar larva of Oxyagrion sulmatogrossense Costa, Souza \& Santos (Odonata, Coenagrionidae)}

Abstract. The last instar larva of Oxyagrion sulmatogrossense Costa, Souza \& Santos 2000 is described and illustrated based on material collected in Cascavel Municipality, Paraná State, Brazil.

Keywords: Immatures, Morphology, Systematic, Zygoptera

$\mathbf{O}$ gênero Oxyagrion Selys, 1876 inclui 23 espécies com ocorrência sulamericana (SCHORR et al., 2009), sendo que dessas, 18 ocorrem no Brasil (PAULSON, 2009). Das espécies conhecidas, 14 possuem suas larvas descritas: Oxyagrion basale Selys, 1876; Oxyagrion bruchi Navás, 1924; Oxyagrion chapadense Costa, 1978; Oxyagrion evanescens Calvert, 1909; Oxyagrion haematinum Selys, 1876; Oxyagrion hempeli Calvert, 1909; Oxyagrion impunctatum Calvert, 1909; Oxyagrion microstigma Selys, 1876; Oxyagrion pavidum Hagen in Selys, 1876; Oxyagrion rubidum (Rambur, 1842); Oxyagrion santosi Martins, 1967; Oxyagrion simile Costa, 1978; Oxyagrion sulinum Costa, 1978 e Oxyagrion terminale Selys, 1876. Este trabalho amplia o conhecimento sobre os imaturos, com a descrição da larva de último ínstar de Oxyagrion sulmatogrossense Costa, Souza \& Santos, 2000.

\section{MATERIAL E MÉTODOS}

As larvas de Oxyagrion sulmatogrossense foram coletadas no Lago Municipal de Cascavel e no distrito de Juvinópolis, Município de Cascavel, Estado do Paraná, Brasil. As coletas aconteceram no período de março de 2006 a janeiro de 2007, resultando em 55 exemplares de imaturos da espécie. Os imaturos de último estádio foram criados em laboratório até a emergência dos adultos. Foram examinadas as 53 exúvias, sendo 29 provenientes de machos e 24, de fêmeas. A identificação foi feita a partir de caracteres dos adultos com uso dos trabalhos de COSTA, SOUZA \& OLDRINI (2004) e LENCIONI (2006) para o gênero e de SANTOS (1966); BULLA (1973) e COSTA, SOUZA \& SANTOS (2000) para a espécie. As medidas foram tomadas sob microscópio esteroscópio Olympus SZX16 e estão expressas em milímetros. Foram tomadas sobre dez exemplares aleatórios, sendo representados os limites mínimos e máximos observados. As ilustrações foram elaboradas a partir de fotografias obtidas com equipamentos acoplados ao estereoscópico e então elaboradas com ajuda do software Adobe Illustrator $\mathrm{CS}_{3}{ }^{\circledR}$. O material está depositado na coleção da Universidade Estadual do Oeste do Paraná, campus de Cascavel.

\section{RESULTADOS}

Oxyagrion sulmatogrossense Costa, Souza \& Santos 2000

Medidas. Comprimento total (excluindo lamela caudal): 10,20/12,90; comprimento da cabeça: 1,35/2,28; largura máxima da cabeça: $2,64 / 3,28$; comprimento dos antenômeros: I: $0,23 / 0,43$; II: $0,40 / 0,48$; III: $0,43 / 0,53$; IV: $0,25 / 0,40$; V: o,23/o,30; VI: o,15/o,20; VII: 0o,5/o,10; comprimento do lábio: 2,18/2,76; largura máxima do lábio: 1,51/2,00; comprimento da lamela caudal: 5,00/6,70; largura máxima da lamela caudal: $0,80 / 1,48$.

Descrição. Coloração geral ocrácea, com antena enegrecida nos $1 / 4$ basal do terceiro antenômero, 3/4 basal do quarto antenômero e $1 / 2$ basal do quinto antenômero, e também com máculas pontilhadas no occipício. Tórax ocráceo, com mácula pontual próxima a articulação com as coxas. Tergitos marrom claro, mais escurecidos lateralmente a linha mediana a partir do quarto segmento. Esternitos marrom claro, mais escurecidos na margem distal de todos os segmentos.

Cabeça mais larga que longa, com mais de 30 setas espiniformes no bordo occipital (Figura 1). Na superfície ventral, uma fileira com sete espinhos, que aumentam e diminuem gradualmente, formando um arco convexo (Figura 1). Antena com sete antenômeros, sendo os dois primeiros mais robusto e o terceiro mais longo que os demais.

Lábio triangular, mais longo que largo, alcançando anteriormente o nível do segundo par de coxas, quando em repouso. Premento (Figura 3) com três setas primárias e uma secundária. Bordo lateral com 10 cerdas espiniformes e articulação do palpo labial com três setas de mesma forma e tamanho (Figura 3). Margem distal levemente crenulada. Palpo labial (Figura 2) 
com cinco setas. Bordo distal com cinco dentículos próximos à garra móvel, seguidos de três dentes triangulares e um dente maior, afilado e curvado para baixo. Margem externa do palpo com cinco setas.

Tórax ocráceo, com margem lateral do protórax com um tubérculo. Sintórax ocráceo, pernas curtas, sendo que as pernas medianas atingem o sexto segmento abdominal. Fêmures e tíbias com espinhos nas margens. Tecas alares estendendo até a margem do terceiro segmento abdominal.

Abdômen cilíndrico, com carena lateral bem desenvolvida nas pleuras do segundo e décimo segmentos. Nono segmento do macho com urômeros curtos, alcançando o terço basal do décimo segmento, com fileira de espinhos ao longo do bordo externo do mesmo (Figura 5). Ovipositor e urômeros da fêmea longos, ultrapassando o décimo segmento abdominal. Urômeros com uma fileira de espinhos que se estendem pelos 3/4 basais do mesmo (Figura 6). Décimo segmento abdominal com uma fileira de pequenos espinhos interrompidos na área basal dos apêndices laterais (Figuras 7 e 8). Cercos curtos, $m$ forma de cone em vista lateral. (Figuras 5 e 6 ). Lamela caudal foliácea pigmentada (Figura 4), com traquéias secundárias e sem sutura transversa. A traquéia principal é espessa. Carina lateral e dorsal da lamela com pequenos espinhos na metade proximal. Lamela mais larga na metade distal.

Material examinado. Brasil. Paraná: Cascavel (Lago Municipal): 31.iii.2006 (emergência 07.iv.2006) uma fêmea, G.S. Andrade, M. E. Rodrigues \& M.S. Dalzochio leg.; 31.iii.2006 (emergência 12.iv.2006) um macho, G.S. Andrade, M. E. Rodrigues \& M.S. Dalzochio leg.; 28.viii.2006 (emergência 13.ix.2006) uma fêmea, G.S. Andrade, M. E. Rodrigues \& M.S. Dalzochio leg.; 13.ix.2006 (emergência 16.ix.2006) uma fêmea, G.S. Andrade, M. E. Rodrigues \& M.S. Dalzochio leg.; 13.ix.2006 (emergência 22.ix.2006) uma femea, G.S. Andrade, M. E. Rodrigues \& M.S. Dalzochio leg.; 13.ix.2006 (emergência 27.ix.2006) uma fêmea, G.S. Andrade, M. E. Rodrigues \& M.S. Dalzochio leg.; 05.x.2006 (emergência 13.x.2006) uma fêmea, G.S. Andrade, M. E. Rodrigues \& M.S. Dalzochio leg.; 05.x.2006 (emergência 28.x.2006) um macho, G.S.Andrade, M.E. Rodrigues \& M.S. Dalzochio leg.; 18.x.2006 (emergência 20.x.2006) um macho, G.S. Andrade, M. E. Rodrigues \& M.S. Dalzochio leg.; 18.x.2006 (emergência 30.x.2006) um macho, G.S. Andrade, M. E. Rodrigues \& M.S. Dalzochio leg.; 18.x.2006 (emergência 06.xi.2006) um macho, G.S. Andrade, M. E. Rodrigues \& M.S. Dalzochio leg.; 16.xi.2006 (emergência 17.xi.2006) uma fêmea, G.S. Andrade, M. E. Rodrigues \& M.S. Dalzochio leg.; 16.xi.2006 (emergência17.xi.2006) um macho, G.S.Andrade,M.E. Rodrigues \& M.S. Dalzochio leg.; 16.xi.2006 (emergência 25.xi.2006) um macho, G.S. Andrade, M. E. Rodrigues \& M.S. Dalzochio leg.; 16.xi.2006 (emergência 29.xi.2006) dois machos, G.S. Andrade, M. E. Rodrigues \& M.S. Dalzochio leg.; 16.xi.2006 (emergência 04.xii.2006) um macho, G.S. Andrade, M. E. Rodrigues \& M.S. Dalzochio leg.; 07.xii.2006 (emergência 08.xii.2006) uma fêmea, G.S. Andrade, M. E. Rodrigues \& M.S. Dalzochio leg.; 07.xii.2006 (emergência 08.xii.2006) uma fêmea, G.S. Andrade, M. E. Rodrigues \& M.S. Dalzochio leg.; 07.xii.2006 (emergência 09.xii.2006) uma fêmea, G.S. Andrade, M. E. Rodrigues \& M.S. Dalzochio leg.; 07.xii.2006 (emergência 12.xii.2006) duas fêmeas, G.S. Andrade, M. E. Rodrigues \& M.S. Dalzochio leg.; 07.xii.2006 (emergência 15.xii.2006) uma fêmea, G.S. Andrade, M. E. Rodrigues \& M.S. Dalzochio leg.; 09.i.2007 (emergência 09.i.2007) uma fêmea, G.S. Andrade, M. E. Rodrigues \& M.S. Dalzochio leg.; 09.i.2007 (emergência 09.i.2007) um macho, G.S. Andrade, M. E. Rodrigues \& M.S. Dalzochio leg.; 09.i.2007 (emergência 12.i.2007) um macho, G.S. Andrade, M. E. Rodrigues \& M.S. Dalzochio leg.; 09.i.2007 (emergência 14.i.2007) um macho, G.S. Andrade, M. E. Rodrigues \& M.S. Dalzochio leg.; 09.i.2007 (emergência 15.i.2007) um macho, G.S. Andrade, M. E. Rodrigues \& M.S. Dalzochio leg.; 09.i.2007 (emergência 22.i.2007) um macho, G.S. Andrade, Andrade, M. E. Rodrigues
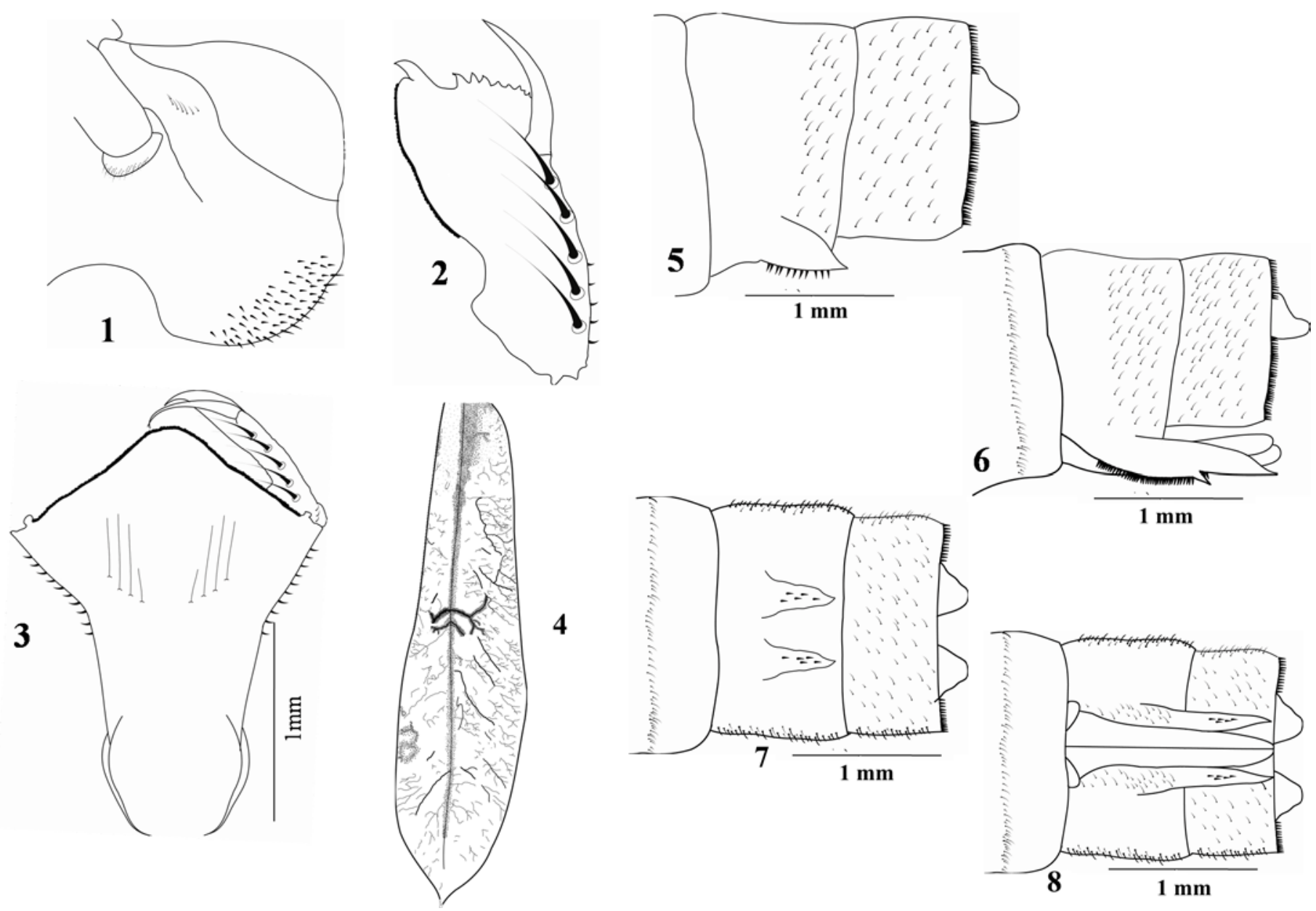

Figuras 1- 8: Oxyagrion sulmatogrossense: (1) Cabeça, vista ventral; (2) palpos labiais, vista interna; (3) lábio, vista interna. (4) lamela caudal, vista lateral; (5) segmentos terminais e cercos do macho, vista lateral; (6) segmentos terminais e cercos da fêmea, vista lateral; (7) segmentos terminais e cercos do macho, vista ventral e; (8) segmentos terminais e cercos da fêmea, vista ventral. 
\& M.S. Dalzochio leg. Distrito de Juvinópolis (Sitio Rodrigues): 02.iv.2006 (emergência 04.iv.2006) um macho, G.S. Andrade, M. E. Rodrigues \& M.S. Dalzochio leg.; 02.iv.2006 (emergência 04.iv.2006) uma fêmea, G.S. Andrade, M. E. Rodrigues \& M.S. Dalzochio leg.; 02.iv.2006 (emergência 05.iv.2006) uma fêmea, G.S. Andrade, M. E. Rodrigues \& M.S. Dalzochio leg.; 02.iv.2006 (emergência 08.iv.2006) um macho, G.S. Andrade, M. E. Rodrigues \& M.S. Dalzochio leg.; 02.iv.2006 (emergência 10.iv.2006) um macho, G.S. Andrade, M. E. Rodrigues \& M.S. Dalzochio leg.; 02.iv.2006 (emergência 10.iv.2006) uma fêmea, A.P.G. Silva leg.; 02.iv.2006 (emergência 11.iv.2006) um macho, G.S. Andrade, M. E. Rodrigues \& M.S. Dalzochio leg.; 02.iv.2006 (emergência 11.iv.2006) uma fêmea, G.S. Andrade, M. E. Rodrigues \& M.S. Dalzochio leg.; 02.iv.2006 (emergência 15.iv.2006) uma fêmea, G.S. Andrade, M. E. Rodrigues \& M.S. Dalzochio leg.; 02.iv.2006 (emergência 15.iv.2006) um macho, G.S. Andrade, M. E. Rodrigues \& M.S. Dalzochio leg.; 02.iv.2006 (emergência 17.iv.2006) uma fêmea, G.S. Andrade, M. E. Rodrigues \& M.S. Dalzochio leg.; 02.iv.2006 (emergência 20.iv.2006) duas fêmeas, G.S. Andrade, M. E. Rodrigues \& M.S. Dalzochio leg.; 02.iv.2006 (emergência 20.iv.2006) um macho, G.S. Andrade, M. E. Rodrigues \& M.S. Dalzochio leg.; 18.vi.2006 (emergência 23.vi.2006) um macho, G.S. Andrade, M. E. Rodrigues \& M.S. Dalzochio leg.; 18.vi.2006 (emergência 27.vi.2006) uma fêmea, G.S. Andrade, M. E. Rodrigues \& M.S. Dalzochio leg.; 18.vi.2006 (emergência 03.vii.2006) uma fêmea, G.S. Andrade, M. E. Rodrigues \& M.S. Dalzochio leg.; 18.vi.2006 (emergência 06.vii.2006) um macho, G.S. Andrade, M. E. Rodrigues \& M.S. Dalzochio leg.;18.vi.2006 (emergência 10.vii.2006) uma fêmea, G.S. Andrade, M. E. Rodrigues \& M.S. Dalzochio leg.; 18.vi.2006 (emergência 20.vii.2006) um macho, G.S. Andrade, M. E. Rodrigues \& M.S. Dalzochio leg.; 18.vi.2006 (emergência 27.vii.2006) uma fêmea, G.S. Andrade, M. E. Rodrigues \& M.S. Dalzochio leg.; 18.vi.2006 (emergência 28.vii.2006) um macho, G.S. Andrade, M. E. Rodrigues \& M.S. Dalzochio leg.; 18.vi.2006 (emergência 12.viii.2006) um macho, G.S. Andrade, M. E. Rodrigues \& M.S. Dalzochio leg.; 18.vi.2006 (emergência 13.viii.2006) uma fêmea, G.S. Andrade, M. E. Rodrigues \& M.S. Dalzochio leg.; 18.vi.2006 (emergência o6.x.2006) um macho, G.S. Andrade, M. E. Rodrigues \& M.S. Dalzochio leg.

Comentários. Três exemplares examinados apresentam cinco setas cerdas prementais à esquerda e dois apresentam apenas as três setas cerdas primárias. Três exemplares apresentaram cinco ou seis espinhos na superfície ventral da cabeça.

Habitat. As larvas foram coletadas em lago artificial com alta ação antrópica e em rios de fraca correnteza, associadas à vegetação marginal.

\section{DISCUSSÃO}

A larva de último instar de O. sulmatogrossense parece próxima de O. chapadense por possuir o seguinte conjunto de caracteres: premento com três cerdas primárias e uma secundária, lamela caudal com traquéia secundária evidente e pigmentada. Porém com a avaliação de caracteres diagnósticos propostos na chave de COSTA, SOUZA \& SANTOS (2000), pode-se observar que O. sulmatogrossense separa-se de O. chapadense por possuir bordo inferior dos olhos, próximos à maxila, com sete espinhos e não somente quatro como em $\mathrm{O}$. chapadense, ou seis em $\mathrm{O}$. basale e pela presença de uma fileira de pequenos espinhos no décimo segmento abdominal que se interrompem na área basal dos cercos (Figura 5).

\section{AGRADECIMENTOS}

Ao Conselho Nacional Desenvolvimento Científico e Tecnológico (CNPq), aos Profs. Drs. Luis Francisco Angeli Alves, Gabriel Simões de Andrade (Universidade Estadual do Oeste do Paraná, Cascavel) e Luis Onofre Irineu de Souza (UFMS) pelas facilidades concedidas no desenvolvimento e elaboração deste trabalho.

\section{REFERÊNCIAS BIBLIOGRÁFICAS}

Bulla, L.A., 1973. Cinco ninfas nuevas o poço conocidas del gênero Oxyagrion Selys. Revista del Museu de La Plata (Nueva Serie), Tomo XII, Zoologia, 112: 11-25.

Costa, J.M., L.O.I. Souza \& B.B. Oldrini, 2004. Chave para Identificação das Famílias e Gêneros das larvas de Odonata do Brasil: Comentários e Registros Bibliográficos (Insecta: Odonata). Publicações Avulsas do Museu Nacional, 99: 3-42.

Costa, J. M., L.O.I. Souza \& T.C. Santos, 2000. Two new species of Oxyagrion Selys, 1876, with a description of five new larvae (Zygoptera: Coenagrionidae). Odonatologica, 29: 1-15.

Lencioni, F.A.A., 2006. Damselflies of Brazil. An illustrated identification guide. II Coenagrionidae. São Paulo, All Print Ed., 419p.

Paulson, D.R., 2009. South American Odonata; List of Odonata of South America by contries. Slater Museum of Natural History, University of Pugetsound. Disponível em: http://www.pugetsound.edu/x7039.xml. Acesso em 18.jun.2009.

Santos, N.D., 1966. Notas sobre a ninfa de Oxyagrion brevistigma Selys. Atas da Sociedade Biológica do Rio de Janeiro, 4: 101103.

Schorr, M., M. Lindeboom \& D.R. Paulson, 2009. World Odonata List. Slater Museum of Natural History, University of Pugetsound. Disponível em: http://www.pugetsound.edu/x6140.xml. Acesso em 18.jun.2009.

Recebido em: 16/06/2009

Aceito em: 12/11/2009

$* * * * * * * * * * * * *$

\section{Como citar este artigo:}

Dalzochio, M.S. \& M.É. Rodrigues, 2009. Descrição da larva de último estádio de Oxyagrion sulmatogrossense Costa, Souza \& Santos (Odonata, Coenagrionidae). EntomoBrasilis, 2(3): 73-75. www.periodico.ebras.bio.br/ojs 